\title{
É financeiramente viável ser verde? A relação entre as práticas de divulgação e a performance financeira de empresas brasileiras de alto impacto ambiental*
}

\author{
¿Es financieramente viable ser verde? La relación entre las prácticas de informe ambiental y el desempeño financiero \\ de empresas brasileras de alto impacto ambiental \\ Is it financially feasible to be green? The relationship between disclosure practices and the financial performance of \\ Brazilian companies with high environmental impact
}

\author{
Márcio Nunes Da Silva ${ }^{a}$ \\ Universidade Federal de Pernambuco, Brasil \\ marcio_nunessilva@hotmail.com \\ ORCID: http://orcid.org/0000-0001-6093-1286 \\ Raimundo Nonato Rodrigues \\ Universidade Federal de Pernambuco, Brasil \\ ORCID: http://orcid.org/0000-0003-0505-4369 \\ Umbelina Cravo Teixeira Lagioia \\ Universidade Federal de Pernambuco, Brasil \\ ORCID: http://orcid.org/0000-0001-8422-7808
}

Recepção: 11 Novembro 2018

Aprovação: 13 Maio 2019

Publicado: 30 Junho 2019

DOI: https://doi.org/10.11144/Javeriana.cc20-49.ffgr

\section{Resumo:}

O presente estudo analisa a relação entre as práticas de divulgação ambiental e o desempenho financeiro de empresas de alto impacto ambiental listadas na Bolsa de valores brasileira. Foi selecionada uma amostra de todas as empresas de alto impacto, de acordo com o Relatório de sustentabilidade de 2016 da Global Reporting Initiative. 34 indicadores ambientais foram correlacionados com três indicadores financeiros (crescimento das vendas, o retorno sobre o patrimônio líquido e o fluxo de caixa operacional / receitas). Enquanto algumas subcategorias ambientais mostraram uma correlação positiva com os indicadores financeiros, outras revelaram correlações negativas. Além disso, os resultados demonstraram que a maioria dos indicadores não apresentaram correlações significativas. Isto pode indicar a necessidade de ações governamentais e corporativas mais efetivas no intuito de se alcançar um desempenho mais sustentável das empresas brasileiras.

Códigos JEL: M49, Q56

Palavras-chave: Divulgação Ambiental, GRI, Teoria da legitimidade, Teoria do disclosure ambiental, contabilidade, auditoria.

\section{Resumen:}

El presente estudio analiza la relación entre las prácticas de divulgación ambiental y el desempeño financiero de empresas de alto impacto listadas en la Bolsa de valores brasilera. Fue seleccionada una muestra de todas las empresas de alto impacto, de acuerdo con el Informe de sustentabilidad de 2016 de la Global Reporting Initiative. 34 indicadores ambientales fueron correlacionados con tres indicadores financieros (crecimiento de ventas, retorno sobre el patrimonio líquido y flujo de caja operacional / ingresos). Mientras algunas subcategorías ambientales mostraron una correlación positiva con los indicadores financieros, otras revelaron correlaciones negativas. Además, los resultados demostraron que la mayoría de los indicadores no presentaron correlaciones significativas. Esto puede indicar la necesidad de acciones gubernamentales y corporativas más efectivas, con el objeto de alcanzar un desempeño más sustentable de las empresas brasileras.

Códigos JEL: M49, Q56

Palabras clave: Divulgación Ambiental, GRI, teoría de la legitimidad, teoría de la divulgación ambiental, contabilidad, auditoria.

\section{Abstract:}

This study analyzes the relationship between environmental disclosure practices and the financial performance of companies with high environmental impact listed on the Brazilian stock exchange. A sample of all high-impact companies was selected, according to the 2016 Global Reporting Initiative. 34 environmental indicators were correlated with three financial indicators (sales

\section{Autor notes:}

\footnotetext{
a Autor correspondente. Correio eletrônico: marcio_nunessilva@hotmail.com
} 
growth, return on equity and operating cash flow / revenues). While some environmental subcategories showed a positive correlation with the financial indicators, some others do not. Additionally, the results showed that most of indicators do not present significative correlations. That would suggest the necessity of more effective governmental and corporate policies to achieve a more sustainable performance of Brazilian companies.

JEL Codes: M49, Q56

Keywords: Environmental Disclosure, GRI, Theory of legitimacy, Theory of environmental disclosure, accounting, auditing.

\section{INTRODUÇÃO}

O debate sobre as questões ambientais e sociais está crescendo ao longo dos anos, não apenas no mundo dos negócios e na sociedade, mas também no meio acadêmico. As empresas estão, cada vez mais, sob pressão quanto ao fornecimento de relatórios de sustentabilidade do impacto social e ambiental de suas atividades. Aquelas que agridem ao meio ambiente e não respeitam as questões sociais, acostumam não ser bem vistas pela sociedade (Deegan, 2002).

Neste âmbito, normas e diretrizes sobre sustentabilidade foram criadas ao longo dos anos (Knebel \& Seele, 2015). A Global Reporting Initiative -GRI- é uma organização internacional independente que foi pioneira em relatórios de sustentabilidade desde 1997. Ela contribui para que as empresas e os governos de todo o mundo compreendam e comuniquem seus impactos ambientais, sociais e econômicos. Deste modo, a GRI procura que essas informações inspirem responsabilidade, ajudem a identificar e gerenciar riscos e permitam que as organizações aproveitem novas oportunidades.

Em se tratando, especificamente, das informações ambientais, há uma discussão na literatura sobre a relação entre as práticas de divulgação ambiental e a performance financeira das organizações (Aggarwal, 2013; Dobre, Stanila \& Brad, 2015; Montabon, Sroufe \& Narasimhan, 2007). Enquanto alguns estudos evidenciaram uma correlação positiva (Lo, Yeung \& Cheng, 2012), outros não encontraram correlações significativas (Chen, Tang \& Feldmann, 2015; Klassen \& McLaughlin, 1996; Russo \& Fouts, 1997).

Além disso, algumas pesquisas evidenciaram relações positivas e negativas a depender de cada indicador analisado (Itawa \& Okada, 2011). Uma das explicações para essa variação de resultados é a falta de medidas objetivas para o desempenho ambiental e para os relatórios de sustentabilidade (Moneva \& Cuellar, 2009).

Na prática, diversas empresas são pressionadas, pelos seus stakeholders, a serem socialmente responsáveis e a respeitarem o meio ambiente (Hofer, Cantor \& Dai, 2012). Entretanto, é importante se perguntar como estes custos ambientais impactam no desempenho financeiro das organizações. De acordo com Chen, Tang e Feldmann (2015), as empresas estão tendo a percepção comum de que a gestão verde aumenta os custos e reduz os lucros, o que desencoraja o esforço para as práticas de divulgação ambiental. Por outro lado, as empresas, sobretudo as de alto impacto ambiental, precisam divulgar mais informações ambientais que as demais organizações de médio e baixo impacto, pois precisam se legitimar perante à sociedade (Silva et al., 2015).

De acordo com Chen, Tang e Feldmann (2015), ainda há uma quantidade limitada de pesquisa que explora a relação entre o desempenho ambiental e o desempenho financeiro das empresas. Portanto, esta pesquisa contribui para ampliar o conhecimento sobre performance financeira e sua relação com as divulgações ambientais de empresas de alto impacto no contexto brasileiro. Pesquisas deste tipo vão além da análise do comportamento dessas entidades. Elas envolvem também interesses sociais e governamentais. De acordo com Itawa e Okada (2011), se há uma relação positiva entre o desempenho ambiental e o desempenho financeiro, as empresas serão incentivadas a reduzir os danos ao meio ambiente, e o governo não precisaria interferir nestas questões.

Nesse contexto, o presente estudo analisa a relação entre as práticas de divulgação ambiental e o desempenho financeiro das empresas de alto impacto ambiental listadas na bolsa de valores brasileira (B3). A 
seguir, é apresentada uma revisão da literatura sobre o assunto; na sequência serão discutidos a metodologia, a análise dos resultados e as conclusões.

\section{REFERENCIAL TEÓRICO}

\section{A Teoria do disclosure ambiental voluntário e a teoria da legitimidade}

Algumas perspectivas teóricas oferecem explicações sobre os motivos pelos quais as empresas optam por divulgarem publicamente informações sobre seu desempenho social ou ambiental. Entre elas, pode se ressaltar a teoria do disclosure voluntário, a teoria da legitimidade e a hipótese dos custos políticos (Adams et al., 1998; Deegan, 2002; Deegan \& Gordon, 1996; Deegan \& Rankin, 1997; Guthrie \& Parker, 1989; Hummel \& Schlick, 2016; Patten, 1992; Roberts, 1992).

A teoria do disclosure ambiental voluntário defende que o objetivo da divulgação voluntária não é apenas fornecer informações sobre as atividades da empresa. Ela também ajuda às empresas na resposta às pressões sociais e políticas colocadas por partes interessadas como governos, a mídia e a sociedade como um todo (Akbaş \& Canikli, 2018).

Segundo a teoria a legitimidade, a relação entre a sociedade e as organizações é estabelecida por meio de um contrato social que poderá ser revogado caso a entidade o viole (Deegan, 2002). As consequências da violação podem afetar a sobrevivência das empresas. Portanto, elas utilizam os relatórios de sustentabilidade para se legitimarem perante os stakeholders (Adams, 2002; Ferreira Neto et al., 2017; Hummel \& Schlick, 2016; Patten, Ren \& Zhao, 2015).

Cho et al. (2015) argumentam que as pressões sociais e institucionais contraditórias exigem que as organizações se envolvam em hipocrisia e desenvolvam "fachadas" (façades). Deste modo, se limitam as perspectivas de que os relatórios de sustentabilidade evoluam de modo substantivo. Os autores defendem o uso de conceitos como hipocrisia e fachada organizacional na literatura de sustentabilidade, porque eles fornecem espaço teórico para debater e refletir sobre o impacto das demandas dos stakeholders nas escolhas das corporações relativas às questões sociais e ambientais.

Por sua vez, Ferreira Neto et al. (2017) investigaram o impacto que os desastres ambientais têm sobre o volume de divulgação e os investimentos socioambientais das empresas brasileiras. Os resultados da pesquisa apontaram que as empresas divulgaram maior volume de informação socioambiental nos dois anos após a ocorrência dos acidentes, o que está de acordo com os preceitos da teoria da legitimidade.

Segundo Hummel e Schlick (2016), apesar das teorias do disclosure e da legitimidade parecerem contraditórias, Elas fazem parte de dois lados opostos de uma mesma moeda. Os autores afirmam que consistente com a teoria do disclosure voluntário, as empresas que possuem uma alta performance na sustentabilidade optam por uma divulgação ambiental de alta qualidade, no intuito de sinalizar seu bom desempenho ao mercado. Em contrapartida, com base na teoria da legitimidade, os atores com uma baixa performance de sustentabilidade preferem uma divulgação de baixa qualidade para disfarçar seu verdadeiro desempenho e, simultaneamente, proteger sua legitimidade.

\section{As práticas de divulgação ambiental e a performance financeira}

A conscientização ambiental dos consumidores, organizações não-governamentais, fornecedores e acionistas, entre outros stakeholders, tem crescido nos últimos anos. Segundo Itawa e Okada (2011), a conscientização e cobrança por um bom desempenho ambiental influenciam direta ou indiretamente o desempenho financeiro das empresas. Portanto, se uma empresa provoca danos irreparáveis ao meio ambiente, ela sofrerá graves sanções por meio de pagamento de multas, perda de confiança, boicote de mercadorias e serviços. Por outro 
lado, uma entidade que se preocupa constantemente com o meio ambiente, promovendo ações que reduzam seus impactos ambientais pode impulsionar positivamente sua reputação entre os consumidores, investidores e governo.

Entretanto, como pode ser visto na tabela 1, os estudos sobre a relação entre divulgação ambiental e desempenho financeiro apresentaram resultados controversos. Enquanto alguns estudos mostraram que existe uma correlação positiva entre a divulgação ambiental e o desempenho financeiro (Lo, Yeung \& Cheng, 2012), outros estudos parecem nega-la (Chen, Tang \& Feldmann, 2015; Klassen \& McLaughlin, 1996; Russo $\&$ Fouts, 1997). Além disso, alguns estudos encontraram diferentes correlações para cada indicador (Iwata \& Okada, 2011). 
TABELA 1

Revisão da literatura

\begin{tabular}{|c|c|c|c|c|}
\hline Título & Ano & Autor & Objetivo & Resultados \\
\hline $\begin{array}{l}\text { Does it pay to be } \\
\text { green? an } \\
\text { empirical } \\
\text { examination of } \\
\text { the relationship } \\
\text { between emission } \\
\text { reduction and } \\
\text { firm performance }\end{array}$ & 1996 & $\begin{array}{l}\text { Stuart L. } \\
\text { Hart, } \\
\text { Gautam } \\
\text { Ahuja }\end{array}$ & $\begin{array}{l}\text { Investigar a relação } \\
\text { entre a redução de } \\
\text { emissões de gases } \\
\text { poluentes e o } \\
\text { desempenho da } \\
\text { empresa. }\end{array}$ & $\begin{array}{l}\text { Os resultados indicam } \\
\text { que os esforços para } \\
\text { evitar a poluição e } \\
\text { reduzir as emissões } \\
\text { caem para a 'linha de } \\
\text { fundo' dentro de uma } \\
\text { dois anos de iniciação e } \\
\text { que as empresas com } \\
\text { os mais altos niveis de } \\
\text { emissão são as que } \\
\text { mais ganham. }\end{array}$ \\
\hline $\begin{array}{l}\text { Environmentally } \\
\text { sensitive } \\
\text { disclosures and } \\
\text { financial } \\
\text { performance in a } \\
\text { European setting }\end{array}$ & 2010 & $\begin{array}{l}\text { Voicu D. } \\
\text { Dragomir }\end{array}$ & $\begin{array}{l}\text { O objetivo deste } \\
\text { trabalho foi traçar um } \\
\text { relacionamento, } \\
\text { envolvendo } \\
\text { divulgação ambiental, } \\
\text { desempenho } \\
\text { ambiental e } \\
\text { desempenho } \\
\text { financeiro de } \\
\text { atividades } \\
\text { ambientalmente } \\
\text { sensiveis de empresas } \\
\text { em um cenário } \\
\text { europeu. }\end{array}$ & $\begin{array}{l}\text { Nenhuma associação } \\
\text { foi encontrada entre o } \\
\text { desempenho ambiental } \\
\text { e desempenho } \\
\text { financeiro. }\end{array}$ \\
\hline $\begin{array}{l}\text { How does } \\
\text { environmental } \\
\text { performance } \\
\text { affect financial } \\
\text { performance? } \\
\text { Evidence from } \\
\text { Japanese } \\
\text { manufacturing } \\
\text { firms }\end{array}$ & 2011 & $\begin{array}{l}\text { Hiroki } \\
\text { Iwata, } \\
\text { Keisuke } \\
\text { Okada }\end{array}$ & $\begin{array}{l}\text { Este artigo examina os } \\
\text { efeitos do } \\
\text { desempenho } \\
\text { ambiental no } \\
\text { desempenho } \\
\text { financeiro usando os } \\
\text { dados de Empresas } \\
\text { manufatureiras } \\
\text { japonesas de } 2004 \text { a } \\
2008 \text {. }\end{array}$ & $\begin{array}{l}\text { Nossos resultados de } \\
\text { estimativa mostram os } \\
\text { diferentes efeitos de } \\
\text { cada desempenho } \\
\text { ambiental no } \\
\text { desempenho } \\
\text { financeiro. As emissões } \\
\text { de resíduos geralmente } \\
\text { não apresentam efeitos } \\
\text { significativos sobre o } \\
\text { desempenho } \\
\text { financeiro. Por outro } \\
\text { lado, a redução de } \\
\text { gases de efeito estufa } \\
\text { leva a um aumento no } \\
\text { desempenho financeiro } \\
\text { em toda a amostra e } \\
\text { nas indústrias limpas, } \\
\text { embora não tenha } \\
\text { efeitos significativos } \\
\text { sobre o desempenho } \\
\text { financeiro em } \\
\text { indústrias sujas. }\end{array}$ \\
\hline $\begin{array}{l}\text { Relationship } \\
\text { between } \\
\text { environmental } \\
\text { responsibility and } \\
\text { financial } \\
\text { performance of } \\
\text { firm: A Literature } \\
\text { review }\end{array}$ & 2013 & $\begin{array}{l}\text { Priyanka } \\
\text { Aggarwal }\end{array}$ & $\begin{array}{l}\text { O objetivo deste } \\
\text { estudo é analisar a } \\
\text { relação entre } \\
\text { responsabilidade } \\
\text { ambiental e } \\
\text { desempenho } \\
\text { financeiro da empresa } \\
\text { através da revisão da } \\
\text { literatura existente, de } \\
\text { modo a encontrar } \\
\text { resposta para a } \\
\text { pergunta de pesquisa } \\
\text { "se ser verdé é } \\
\text { rentável para empresa } \\
\text { ou não". }\end{array}$ & $\begin{array}{l}\text { Observou-se que a } \\
\text { maioria dos estudos } \\
\text { indica relação positiva } \\
\text { entre responsabilidade } \\
\text { ambiental e } \\
\text { desempenho } \\
\text { financeiro. Este } \\
\text { trabalho tenta analisar } \\
\text { criticamente estudos } \\
\text { prévios, a fim de } \\
\text { construir escopo para } \\
\text { futuras pesquisas, } \\
\text { para que futuros } \\
\text { pesquisadores possam } \\
\text { alcançar resultados } \\
\text { melhores e mais } \\
\text { consistentes. }\end{array}$ \\
\hline $\begin{array}{l}\text { Applying GRI } \\
\text { reports for the } \\
\text { investigation of } \\
\text { environmental } \\
\text { management } \\
\text { practices and } \\
\text { company } \\
\text { performance in } \\
\text { Sweden, China } \\
\text { and India }\end{array}$ & 2015 & $\begin{array}{l}\text { Lujie Chen, } \\
\text { Ou Tang, } \\
\text { Andreas } \\
\text { Feldmann }\end{array}$ & $\begin{array}{l}\text { Este artigo investigou } \\
\text { a relação entre a } \\
\text { gestão ambiental } \\
\text { (EMP) e o desempenho } \\
\text { da empresa de } \\
\text { manufatura na } \\
\text { Suécia, China e Índia. }\end{array}$ & $\begin{array}{l}\text { Os resultados } \\
\text { indicaram que a } \\
\text { maioria dos EMPS, } \\
\text { claramente, não tem } \\
\text { uma correlação } \\
\text { positiva como } \\
\text { desempenho } \\
\text { financeiro; isto é, } \\
\text { empregar EMPs não } \\
\text { necessariamente } \\
\text { melhora o desempenho } \\
\text { econômico das } \\
\text { empresas. }\end{array}$ \\
\hline
\end{tabular}

Fonte: elaborada pelos autores. 
Dobre, Stanila e Brad (2015) pesquisaram como as empresas listadas na Romênia relatam indicadores ambientais e sociais, e se isso tem ou não impacto sobre o desempenho financeiro. Os resultados indicaram que o aumento da proteção ambiental e social pode ter impacto no desempenho financeiro no longo prazo.

Por sua parte, Simionescu e Dumitrescu (2018) investigaram a relação entre responsabilidade social corporativa (RSC) e o desempenho financeiro (DF) das empresas listadas na Bolsa de Bucareste. Após estimar modelos de regressão de corte transversal, o estudo forneceu suporte para uma relação positiva entre RSC e o DF. Isto se dá quando as empresas implementam políticas de RSC em relação aos empregados, à proteção ambiental, e à ética como práticas sociais.

O estudo realizado por Alexopoulos, Kounetas e Tzelepis (2018) examina a relação entre desempenho ambiental e financeiro em empresas gregas de manufatura. A pesquisa evidenciou que as diferenças nas configurações institucionais, sociais e culturais influenciam o desempenho financeiro das empresas. Essas características parecem limitar os benefícios financeiros dos projetos de melhoria do desempenho ambiental. Apenas as empresas com recursos estão dispostas a tomar as medidas necessárias para os métodos de produção mais "verdes".

\section{METODOLOGIA}

Nesta seção, serão detalhados os procedimentos para a coleta dos dados, a definição da amostra, os critérios para a escolha das variáveis adotadas e a ferramenta para análise dos dados.

\section{Coleta dos dados e Amostra}

O estudo foi realizado com as empresas listadas na B3 que são classificadas como "organizações de alto impacto ambiental". Elas divulgaram o seu relatório de sustentabilidade com base no GRI- G4 referente ao ano de 2016. As empresas de alto impacto foram classificadas de acordo com a lei $\mathrm{n}^{\circ} 6.938 / 81$, atualizada pela lei ${ }^{\circ} 10.165 / 00$. Na tabela 2 pode-se observar todas as empresas que compuseram a amostra. 
TABELA 2

Empresas de alto impacto ambiental por setor

\begin{tabular}{|l|c|}
\hline Minerais metálicos & \\
\hline Vale S.A. & $\begin{array}{c}\text { Extração e tratamento } \\
\text { de minerais }\end{array}$ \\
\hline $\begin{array}{l}\text { Petróleo. Gás e biocombustíveis: exploração. Refino e } \\
\text { distribuição }\end{array}$ & \\
\hline Cosan S.A. & Indústria de papel e \\
Óleo e gás participações S.A. & Celulose
\end{tabular}

Fonte: elaborada pelos autores com base na lei $n^{\circ} 6.938 / 81$, atualizada pela lei $\mathrm{n}^{\circ} 10.165 / 00$.

A GPC participações S.A., Paranapanema S.A. e a Gerdau S.A. foram excluídas da amostra por não apresentarem um relatório de sustentabilidade baseado na GRI- G4 referente ao ano de 2016. Embora a Natura Cosméticos tenha divulgado um relatório baseado no GRI, a empresa utilizou a nova versão, o GRI Standard, portanto a empresa também foi excluída da amostra. Todos os relatórios de sustentabilidade foram baixados por meio de links contidos em uma planilha eletrônica fornecida pelo GRI (reportregistration@globalreporting.org).A seguir, se definem quais foram os indicadores utilizados e como as correlações entre indicadores ambientais e financeiros foram realizadas.

\section{Indicadores ambientais e a análise de conteúdo}

Em cada relatório, das 18 empresas selecionadas, foram analisados os 34 indicadores ambientais do GRIG4, listados no Apêndice 1. O método de análise adotado para os relatórios de sustentabilidade foi a análise de conteúdo, na mesma orientação de estudos similares (Chen, Tang \& Feldmann, 2015; Dragomir, 2010). Para mensurar o desempenho ambiental de cada organização, foi criado um índice de divulgação ambiental baseado nos estudos de Dragomir (2010) e Chen, Feldmann e Tang (2015). Portanto, os indicadores ambientais de cada relatório foram analisados após a leitura, e receberam uma nota de 1 a 5 , de acordo com os critérios descritos da tabela 3. 
TABELA 3

Escala de pontuação para os indicadores ambientais

\begin{tabular}{|l|c|}
\hline $\begin{array}{l}\text { Os dados de desempenho ambiental não estão presentes } \\
\text { (incluindo referências não quantitativas a desempenho). }\end{array}$ & 1 \\
\hline $\begin{array}{l}\text { Informações sobre desempenho e lou governança são } \\
\text { apresentadas apenas para o periodo atual }\end{array}$ & 2 \\
\hline $\begin{array}{l}\text { O relatório e as informações nele contidas podem ser comparados } \\
\text { ano a ano }\end{array}$ & 3 \\
\hline $\begin{array}{l}\text { Os critérios acima, mais as técnicas de mensuração de dados e } \\
\text { bases para cálculos são adequadamente descritos e podem ser } \\
\text { replicados com resultados semelhantes. }\end{array}$ & 4 \\
\hline $\begin{array}{l}\text { Os critérios acima, além do desempenho da organização, podem } \\
\text { ser comparados com benchmarks apropriados. }\end{array}$ & 5 \\
\hline
\end{tabular}

Fonte: Dragomir (2010) e Chen, Feldmann e Tang (2015).

Enquanto o estudo de Dragomir (2010) adotou uma escala de 0 a 4, este artigo empregou uma escala de 1 a 5, de acordo com Chen, Feldmann e Tang (2015). A pontuação da escala utilizada aumenta de acordo com o aumento da qualidade das informações apresentadas nos relatórios de sustentabilidade. Como a pesquisa se propõe a analisar a relação entre as práticas de divulgação ambiental e o desempenho financeiro das empresas, torna-se necessário definir quais foram os indicadores utilizados e como as correlações foram realizadas.

\section{Indicadores financeiros e a correlação dos dados}

Para mensurar a performance financeira foram adotados os seguintes indicadores: crescimento das vendas, Retorno sobre o patrimônio Líquido (ROE) e o fluxo de caixa operacional/vendas (Chen, Feldmann e Tang, 2015). Os dados utilizados para calcular os indicadores financeiros foram coletados das demonstrações financeiras padronizadas de 2015 e 2016 divulgadas no site da Bolsa de Valores B3, na aba "empresas listadas".

- O indicador Crescimento das vendas (CV) foi calculado considerando a receita com vendas de 2015 (RCV15) e 2016 (RCV16). Ele indica a porcentagem do aumento das vendas no período analisado (2015-2016).

\section{CV $=\underline{\text { RCV16-RCV15 }}$ RCV15}

- O indicador Retorno sobre o Patrimônio líquido (ROE) pode ser analisado como a capacidade que a empresa possui de remunerar os seus proprietários.

$$
\text { ROE }=\frac{\text { Lucro líquido }}{\mathrm{PL}}
$$

- O indicador fluxo de caixa operacional/vendas (FCO/V) mede a capacidade da empresa em transformar receita em caixa operacional.

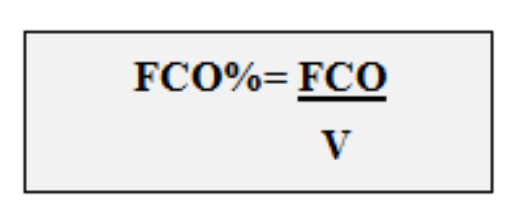


As relações entre os indicadores ambientais e financeiros foram obtidas por meio do coeficiente de correlação de Spearman (CCS). Segundo Field (2009), o CCS é uma medida padronizada da força do relacionamento entre duas variáveis que não depende das hipóteses de um teste paramétrico. Para testar a consistência interna entre as subcategorias, o Alpha de Cronbach foi calculado. Para organizar e analisar os dados foram utilizados, respectivamente, o MS Excel 2016 e o software SPSS Statistics 20.

\section{RESULTADOS}

\section{Análise descritiva}

Ao analisar a estatística descritiva da categoria ambiental, na tabela 4, percebe-se que os indicadores EN15 (Emissões diretas de gases de efeito estufa) e EN3 (Consumo de energia dentro da organização) apresentaram, respectivamente, as maiores médias. Notou-se que os indicadores que apresentam as maiores médias foram aqueles mais facilmente quantificáveis. Os resultados estão de acordo com o trabalho de Chen, Feldmann e Tang (2015). Por outro lado, os indicadores EN28 (Percentual de produtos e suas embalagens recuperados em relação ao total de produtos vendidos discriminado por categorias de produto) e EN2 (Percentual de materiais usados provenientes de reciclagem) foram os menos divulgados. 
TABELA 4

Estatística descritiva sobre a categoria ambiental

\begin{tabular}{lccccc}
\hline & N & MIN & MAX & MÉDIA & DESVIO \\
EN1 & 18 & 1 & 5 & 1,78 & 1,56 \\
EN2 & 18 & 1 & 5 & $\mathbf{1 , 1 7}$ & 0,51 \\
EN3 & 18 & 1 & 5 & $\mathbf{3 , 4 4}$ & 1,76 \\
EN4 & 18 & 1 & 5 & 1,78 & 1,52 \\
EN5 & 18 & 1 & 5 & 2,39 & 1,75 \\
EN6 & 18 & 1 & 5 & 1,78 & 1,44 \\
EN1 & 18 & 1 & 5 & 1,22 & 0,94 \\
EN8 & 18 & 1 & 5 & 2,39 & 1,72 \\
EN9 & 18 & 1 & 5 & 1,22 & 0,55 \\
EN10 & 18 & 1 & 5 & 2,33 & 1,57 \\
EN11 & 18 & 1 & 5 & 1,50 & 0,79 \\
EN12 & 18 & 1 & 5 & 1,72 & 0,75 \\
EN13 & 18 & 1 & 5 & 1,44 & 0,70 \\
EN14 & 18 & 1 & 5 & 1,67 & 0,97 \\
EN15 & 18 & 1 & 5 & $\mathbf{3 , 7 2}$ & 1,67 \\
EN16 & 18 & 1 & 5 & 3,33 & 1,85 \\
EN17 & 18 & 1 & 5 & 2,89 & 1,75 \\
EN18 & 18 & 1 & 5 & 2,78 & 1,83 \\
EN19 & 18 & 1 & 5 & 1,94 & 1,55 \\
EN20 & 18 & 1 & 5 & 1,94 & 1,55 \\
EN21 & 18 & 1 & 5 & 2,17 & 1,54 \\
EN22 & 18 & 1 & 5 & 2,50 & 1,50 \\
EN23 & 18 & 1 & 5 & 2,72 & 1,56 \\
EN24 & 18 & 1 & 5 & 1,56 & 1,04 \\
EN25 & 18 & 1 & 5 & 1,56 & 1,15 \\
EN26 & 18 & 1 & 5 & 2,11 & 1,68 \\
EN27 & 18 & 1 & 5 & 2,11 & 1,68 \\
EN28 & 18 & 1 & 5 & $\mathbf{1}, 06$ & 0,24 \\
EN29 & 18 & 1 & 5 & 1,67 & 0,91 \\
EN30 & 18 & 1 & 5 & 1,89 & 1,18 \\
EN31 & 18 & 1 & 5 & 1,61 & 1,20 \\
EN32 & 18 & 1 & 5 & 1,39 & 0,98 \\
EN33 & 18 & 1 & 5 & 1,28 & 0,96 \\
EN34 & 18 & 1 & 5 & 1,28 & 0,57 \\
\hline & & & 5 & & \\
\hline
\end{tabular}

Fonte: Elaborada pelos autores.

$\mathrm{Na}$ tabela 5 percebe-se que as empresas que apresentaram as maiores médias referentes a divulgação de informações ambientais foram, respectivamente, a QGEP Participações S.A., a BRASKEM S.A. e a VALE S.A. Portanto, essas empresas divulgaram mais informações ambientais que podem ser comparadas ano a ano; detalham mais as técnicas de mensuração de dados e bases para cálculos, as quais servem para replicações ou até mesmo podem ser comparados com benchmarks apropriados. 
TABELA 5

Estatística descritiva sobre os indicadores ambientais das empresas de alto impacto

\begin{tabular}{|c|c|c|c|}
\hline & Total & Media & STD \\
\hline \multicolumn{4}{|c|}{ Minerais Metálicos } \\
\hline Vale S.A. & 93 & 2,74 & 1,44 \\
\hline \multicolumn{4}{|c|}{ Exploração. Refino e distribuição } \\
\hline Cosan S.A. & 45 & 1,32 & 0,91 \\
\hline Oleo e Gás Participações S.A. & 35 & 1,03 & 0,17 \\
\hline Petroleo Brasileiro S.A. & 50 & 1,47 & 1,31 \\
\hline QGEP Participações S.A. & 99 & 2,91 & 1,83 \\
\hline \multicolumn{4}{|c|}{ Papel e Celulose } \\
\hline Celulose Irani S.A. & 71 & 2,09 & 1,60 \\
\hline Fibria Celulose S.A. & 59 & 1,74 & 1,02 \\
\hline Suzano Papel e Celulose S.A. & 83 & 2,44 & 1,13 \\
\hline \multicolumn{4}{|c|}{ Fertilizantes } \\
\hline FERTILIZANTES HERINGER S.A. & 47 & 1,38 & 1,07 \\
\hline \multicolumn{4}{|c|}{ Petroquímico } \\
\hline Braskem S.A. & 98 & 2,88 & 1,98 \\
\hline Elekeiroz S.A. & 76 & 2,24 & 1,78 \\
\hline \multicolumn{4}{|c|}{ Produção de Alcool Etilico, Metanol e Similares } \\
\hline Biosev S.A & 55 & 1,62 & 1,16 \\
\hline Raizen Energia S.A. & 85 & 2,50 & 1,71 \\
\hline \multicolumn{4}{|c|}{ Produção de medicamentos } \\
\hline Ouro Fino Saúde Animal Participações S.A. & 62 & 1,82 & 1,40 \\
\hline Raia Drogasil S.A. & 45 & 1,32 & 0,98 \\
\hline \multicolumn{4}{|c|}{ Transporte Aéreo } \\
\hline Gol Linhas Aereas Inteligentes S.A. & 84 & 2,47 & 1,56 \\
\hline \multicolumn{4}{|c|}{ Transporte Ferroviário } \\
\hline Rumo All & 54 & 1,59 & 0,86 \\
\hline \multicolumn{4}{|c|}{ Transporte Rodoviário } \\
\hline JSL S.A. & 71 & 2,09 & 1,48 \\
\hline
\end{tabular}

Fonte: Elaborada pelos autores.

Por outro lado, a Óleo e Gás Participações S.A. e a Cosan S.A. apresentaram as menores médias. Como as médias se aproximaram a 1, significa que muitos indicadores não foram divulgados e/ou os que foram divulgados receberam uma nota baixa por falta de um maior detalhamento das informações.

\section{Constructo do GRI}

O modelo de relatório sugerido pelo GRI é organizado em categorias principais e subcategorias. Para este estudo, apenas a categoria ambiental foi considerada. De acordo com o modelo GRI- G4, a categoria ambiental possui 12 subcategorias, as quais são formadas por 1 ou mais indicadores (Apêndice 1). Foi necessário calcular se havia consistência interna para cada subcategoria que apresenta mais de um indicador (tabela 6). Dessa forma, o Alpha de Cronbach foi calculado para verificar a consistência interna de cada 
constructo. Segundo Field (2009), um alpha de Cronbach entre 0,7 e 0,8 é aceitável e valores mais baixos indicam que a escala não é confiável. Entretanto, há estudos que aceitam valores acima de 0,6.

TABELA 6

Subcategorias sobre o desempenho ambiental a partir do GRI

\begin{tabular}{llc}
\hline Materiais (EN_A) & EN1, EN2 & $0,595^{*}$ \\
Energia (EN_B) & EN3, EN4, EN5, EN6, EN7 & 0,635 \\
Agua (EN_C) & EN8, EN9, EN10 & 0,727 \\
Biodiversidade (EN_D) & EN11, EN12, EN13, EN14 & 0,739 \\
Emissões (EN_E) & EN15, EN16,EN17, & \\
& EN18,EN19,EN20, EN21 & 0,896 \\
Efluentes e Residuos (EN_F) & EN22, EN23, EN24, EN25, & \\
Produtos e serviços (EN_G) & EN26 & 0,79 \\
Conformidade (EN_H) & EN27, EN28 & $-0,009^{*}$ \\
Transporte (EN_I) & EN29 & - \\
Geral (EN_J) & EN30 & - \\
Avaliação Ambiental de & EN31 & - \\
Fornecedores (EN_K) & & \\
$\begin{array}{l}\text { Mecanismos de Reclamação } \\
\text { Ambiental (EN_L) }\end{array}$ & EN32, EN33 & $0,222^{*}$ \\
\hline
\end{tabular}

Fonte: Baseado em Chen, Feldmann e Tang (2015).

Com base na tabela 6, percebe-se que o Alpha de Cronbach foi menor que 0,6 para as subcategorias Materiais, Produtos e serviços e Avaliação Ambiental de Fornecedores, portanto esses constructos foram excluídos da análise.

\section{Correlações entre as subcategorias ambientais e os indicadores financeiros}

A tabela 7 evidencia as correlações entre as subcategorias ambientais e os indicadores financeiros. É possível notar que o EN_D (Biodiversidade) apresentou correlação positiva com a taxa de crescimento das vendas. Um destaque pode ser dado ao EN12 o qual apresentou a maior correlação $(0,403)$ com o indicador "taxa de crescimento nas vendas" (apêndice 2). Portanto, a divulgação de impactos significativos de atividades, produtos e serviços sobre a biodiversidade tanto em áreas protegidas quanto em áreas não protegidas, apresentou uma relação positiva com o aumento das vendas.

Enquanto que o EN_E (Emissões) apresentou uma correlação positiva com a taxa de crescimento das vendas e uma correlação negativa com o ROE. Entre todos os indicadores que apresentaram uma correlação negativa, o EN15 (Emissões de gases de efeito estufa) se destacou $(-0,608)$. Dessa forma, quanto mais divulgação sobre emissões diretas de gases de efeito estufa, menor foi o retorno sobre o patrimônio líquido, o que está de acordo com o estudo de Hart e Ahuja (1996).

O EN_I (transporte) apresentou uma correlação positiva com a taxa de crescimento das vendas. Esta subcategoria conta com apenas um indicador, o EN30 (Impactos ambientais significativos decorrentes do transporte de produtos e outros bens e materiais usados nas operações da organização, bem como do transporte de seus empregados). Nesse sentido, o relato sobre os impactos ambientais provocados pelo transporte de produtos ou empregados contribuíram para a taxa de crescimento das vendas.

Da mesma forma, o EN_L (Mecanismos de Reclamação Ambiental) apresentou uma correlação positiva com a taxa de crescimento das vendas. O único indicador pertence a esta subcategoria é o EN34 (Número de queixas e reclamaçóes relacionadas a impactos ambientais protocoladas, processadas e solucionadas por meio de mecanismo formal). Portanto, quanto mais divulgação sobre queixas ambientais solucionadas, maiores foram as vendas. 
TABLA 7

Correlações entre as subcategorias ambientais e os indicadores financeiros

\begin{tabular}{|c|c|c|c|c|}
\hline & & $\begin{array}{c}\text { Taxa de Cresc. } \\
\text { Das Vendas }\end{array}$ & ROE & $\begin{array}{c}\text { Fluxo de caixa } \\
\text { operacional / } \\
\text { Receitas } \\
\end{array}$ \\
\hline \multirow[t]{3}{*}{ EN_B } & Correlation Coefficient & $-0,236$ & $-0,085$ & 0,197 \\
\hline & Sig. (1-tailed) & 0,173 & 0,368 & 0,217 \\
\hline & $\mathrm{N}$ & 18 & 18 & 18 \\
\hline \multirow[t]{3}{*}{ EN_C } & Correlation Coefficient & 0,033 & $-0,181$ & $-0,031$ \\
\hline & Sig. (1-tailed) & 0,450 & 0,244 & 0,453 \\
\hline & $\mathrm{N}$ & 17 & 17 & 17 \\
\hline \multirow[t]{3}{*}{ EN_D } & Correlation Coefficient & $0,459^{*}$ & $-0,081$ & 0,302 \\
\hline & Sig. (1-tailed) & 0,028 & 0,374 & 0,111 \\
\hline & $\mathrm{N}$ & 18 & 18 & 18 \\
\hline \multirow[t]{3}{*}{ EN_E } & Correlation Coefficient & $0,465^{2}$ & $-0,446^{2}$ & 0,164 \\
\hline & Sig. (1-tailed) & 0,026 & 0,032 & 0,257 \\
\hline & $\mathrm{N}$ & 18 & 18 & 18 \\
\hline \multirow[t]{3}{*}{ EN_F } & Correlation Coefficient & $-0,266$ & 0,065 & 0,072 \\
\hline & Sig. (1-tailed) & 0,143 & 0,398 & 0,388 \\
\hline & $\mathrm{N}$ & 18 & 18 & 18 \\
\hline \multirow[t]{3}{*}{ EN_H } & Correlation Coefficient & 0,342 & $-0,014$ & $-0,041$ \\
\hline & Sig. (1-tailed) & 0,082 & 0,478 & 0,435 \\
\hline & $\mathrm{N}$ & 18 & 18 & 18 \\
\hline \multirow[t]{3}{*}{ EN_I } & Correlation Coefficient & $0,514^{\circ}$ & 0,230 & 0,157 \\
\hline & Sig. (1-tailed) & 0,015 & 0,179 & 0,267 \\
\hline & $\mathrm{N}$ & 18 & 18 & 18 \\
\hline \multirow[t]{3}{*}{ EN_J } & Correlation Coefficient & 0,233 & $-0,034$ & 0,256 \\
\hline & Sig. (1-tailed) & 0,176 & 0,447 & 0,152 \\
\hline & $\mathrm{N}$ & 18 & 18 & 18 \\
\hline \multirow[t]{3}{*}{ EN_L } & Correlation Coefficient & $0,460^{\circ}$ & $-0,164$ & 0,275 \\
\hline & Sig. (1-tailed) & 0,027 & 0,258 & 0,135 \\
\hline & $\mathrm{N}$ & 18 & 18 & 18 \\
\hline
\end{tabular}

Fonte: Elaborada pelos autores.

${ }^{*}$ Correlation is significant at the 0.05 level ( 1 -tailed).

${ }^{* *}$ Correlation is significant at the 0.01 level (1-tailed).

Entretanto, quando se observa o indicador Fluxo de caixa operacional/receitas, percebe-se que apenas o EN4 e o EN13 possuem correlação positiva (apêndice 2). Portanto, só para esses indicadores, a divulgação do consumo de energia fora da organização, o relato do tamanho e a localização de todas as áreas protegidas de habitats ou áreas restauradas, contribuíram para transformar receitas de vendas em caixa. Por fim, as subcategorias EN_B, EN_C, EN_F, EN_H e EN_J EN13 não apresentaram correlações significativas com os indicadores financeiros adotados no estudo. 


\section{CONCLUSÃO}

Este estudo correlacionou os indicadores de divulgação ambiental e de desempenho financeiro na lista de empresas de alto impacto ambiental da bolsa de valores brasileira. Os resultados da pesquisa apontaram que os indicadores relacionados a emissóes diretas de gases de efeito estufa e ao consumo de energia dentro da organização foram os mais divulgados, respectivamente. Sob o ponto de vista das empresas, as que apresentaram as maiores médias referentes a divulgação de informações ambientais foram, respectivamente, a QGEP Participações S.A., a Braskem S.A. e a Vale S.A.

A teoria da legitimidade e a teoria do disclosure voluntário podem servir como base para explicar os motivos pelos quais algumas empresas divulgaram mais informações ambientais do que outras, assim como a qualidade das informações divulgadas. Uma explicação para a alta divulgação ambiental pode ser a intenção de sinalizar para o mercado o seu bom desempenho ambiental e aumentar seu valor para os acionistas. Entretanto, numa escala de 1 a 5, cada empresa apresentou uma média abaixo de 3, o que pode indicar uma baixa qualidade das informações geradas, disfarçando assim o seu verdadeiro desempenho ambiental.

Foi possível observar, também, que as subcategorias Biodiversidade (EN_D), Emissões (EN_E), Transporte (EN_I) e Mecanismos de Reclamação Ambiental (EN_L), apresentaram uma correlação positiva com o aumento das vendas. Enquanto que a subcategoria Emissões (EN_E) apresentou uma correlação negativa com o ROE. Isto indica um menor retorno sobre o patrimônio líquido quando se aumenta a divulgação sobre emissões de gases poluentes.

$\mathrm{Na}$ análise do indicador Fluxo de caixa operacional/receitas, percebeu-se que divulgar o consumo de energia fora da organização, e relatar o tamanho e a localização de todas as áreas protegidas de habitats ou áreas restauradas, contribuíram para transformar receitas de vendas em caixa.

Entretanto, os resultados mostram que a maioria dos indicadores não apresentaram correlações significativas, o que pode indicar a necessidade de ações governamentais e corporativas mais efetivas no intuito de se alcançar um desempenho mais sustentável das empresas.

Entre as limitações do estudo, pode-se destacar o corte temporal de apenas um ano. Este corte limitado pode incidir para a não captura de informações relevantes. Outro ponto a destacar é a grande quantidade de páginas dos relatórios e a metodologia de pontuação para os indicadores. Sobre o primeiro, é necessário muita atenção e reflexão, o que pode ser prejudicado pela quantidade excessiva de informações. $O$ processo de atribuir nota aos indicadores foi uma tentativa de tornar o estudo mais objetivo. Entretanto, não é possível eliminar totalmente a subjetividade. Por fim, a quantidade de indicadores financeiros poderia ter sido maior, por exemplo, com a inclusão do indicador Tobin’s Q (Chung \& Pruitt, 1994) e Retorno sobre o ativo (Dragomir, 2010).

Como sugestão para pesquisas futuras, podem-se realizar estudos comparativos das correlações de indicadores ambientais e financeiros entre empresas de alto impacto com as de médio e baixo. Além disso, podem ser realizados estudos longitudinais no intuito de analisar um período mais longo, e incorporando outros indicadores financeiros.

\section{REFERENCIAS}

Adams, C. (2002). Internal organizational factors influencing corporate social and ethical reporting: Beyond current theorizing. Accounting, Auditing and Accountability Journal, 15(2), 223-250.

Adams, C., Hill, W.-Y. \& Roberts, C. (1998). Corporate social reporting practices in Western Europe: legitimating corporate behaviour? The British Accounting Review, 30(1), 1-21. doi: https://doi.org/10.1006/bare.1997.0060

Aggarwal, P. (2013). Relationship between environmental responsibility and financial performance of firm: A literature review. Journal of Business and Management, 13(1), 13-22. 
Akbaş, H. \& Canikli, S. (2018). Determinants of voluntary greenhouse gas emission disclosure: An empirical investigation on Turkish firms. Sustainability, 11(1), 107. doi: https://doi.org/10.3390/su11010107

Alexopoulos, I., Kounetas, K. \& Tzelepis, D. (2018). Environmental and financial performance. Is there a win-win or a win-loss situation? Evidence from the Greek manufacturing. Journal of Cleaner Production, 197(1).

Chen, L., Feldmann, A. \& Tang, O. (2015). The relationship between disclosures of corporate social performance and financial performance: Evidences from GRI reports in manufacturing industry. International Journal of Production Economics, 170, 445-456.

Chen, L., Tang, O. \& Feldmann, A. (2015). Applying GRI reports for the investigation of environmental management practices and company performance in Sweden, China and India. Journal of Cleaner Production, 98(1), 36-46.

Cho, C., Laine, M., Roberts, R. \& Rodrigue, M. (2015). Organized hypocrisy, organizational façades, and sustainability reporting. Accounting, Organizations and Society, 40, 78-94. doi: https://doi.org/10.1016/j.aos.2014.12.003.

Chung, K. \& Pruitt, S. (1994). A simple approximation of Tobin's Q. Financial Management, 23(3), 70-74.

Deegan, C. \& Gordon, B. (1996). A study of the environmental disclosure practices of Australian corporations. Accounting and Business Research, 26(3), 187-199. doi: https://doi.org/10.1080/00014788.1996.9729510.

Deegan, C. \& Rankin, M. (1997). The materiality of environmental information to users of annual reports. Accounting, Auditing \& Accountability Journal, 10(4), 562-583. doi: https://doi.org/10.1108/09513579710367485

Deegan, C. (2002). Introduction: The legitimising effect of social and environmental disclosures - A theoretical foundation. Accounting, Auditing \& Accountability Journal, 15(3), 282-311. doi: https://doi.org/10.1 108/095 13570210435852.

Dobre, E., Stanila, G. \& Brad, L. (2015). The influence of environmental and social performance on financial performance: Evidence from Romania's listed entities. Sustainability, 7, 2513-2553. doi: https://doi.org/10.33 90/su7032513.

Dragomir, V. (2010). Environmentally sensitive disclosures and financial performance in a European setting, Journal of Accounting \& Organizational Change, 6(3), 359-388.

Ferreira N., J., da Silva G., S., Bruni, A. \& Dias F., J. (2017). Do Environmental Disasters Impact on the Volume of SocioEnvironmental Investment and Disclosure of Brazilian Companies? Advances in Environmental Accounting \& Management: Social and Environmental Accounting in Brazil, 159-187. doi: https://doi.org/10.1108/ s1479-359820160000006006

Field, A. (2009). Descobrindo a Estatística usando o SPSS. Porto Alegre: Artmed, 2 ed.

Gallego-Alvarez, I., Lozano, M. \& Rodríguez-Rosa, M. (2018). An analysis of the environmental information in international companies according to the new GRI standards. Journal of Cleaner Production 182, 57-66.

Gri (2018). Pioneer of Sustainability Reporting. Disponível em: https://www.globalreporting.org/information/about -gri/Pages/default.aspx (acesso em 01.09.18).

Guthrie, J. \& Parker, L. (1989). Corporate social reporting: A rebuttal of legitimacy theory. Accounting and Business Research, 19(76), 343-352. doi: https://doi.org/10.1080/00014788.1989.9728863.

Hair, J., Anderson, R., Tatham, R. \& Black, W. (1998). Multivariate Data Analysis, 5th ed. Prentice Hall, New Jersey.

Hart, S. \& Ahuja, G. (1996). Does it pay to be green? An empirical examination of the relationship between emission reduction and firm performance. Business Strategy and the Environment, 5, 30-37.

Hofer, C., Cantor, D. \& Dai, J. (2012). The competitive determinants of a firm's environmental management activities: Evidence from US manufacturing industries. Journal Oper. Manag. 30 (1-2), 69-84.

Hummel, K. \& Schlick, C. (2016). The relationship between sustainability performance and sustainability disclosure - Reconciling voluntary disclosure theory and legitimacy theory. Journal of Accounting and Public Policy, 35(5), 455-476. doi: https://doi.org/10.1016/j.jaccpubpol.2016.06.001

Itawa, H. \& Okada, K. (2011). How does environmental performance affect financial performance? Evidence from Japanese manufacturing firms. Ecol. Econ. 2011, 70, 1691-1700.

Klassen, R. \& McLaughlin, C. (1996). The Impact of Environmental Management on Firm Performance. Manag. Sci., $42,1199-1214$. 
Knebel, S. \& Seele, P. (2015). Quo vadis GRI? A (critical) assessment of GRI 3.1 A+ nonfinancial reports and implications for credibility and standardization, Corporate Communications. An International Journal, 20(2), 196-212.

Lo, C., Yeung, A. \& Cheng, T. (2012). The impact of environmental management systems on financial performance in fashions and textile industries. Int.J. Prod. Econ., 135, 561-567.

Magness, V. (2006). Strategic posture, financial performance and environmental disclosure: an empirical test of legitimacy theory. Accounting, Auditing \& Accountability Journal, 19(4), 540-563.

Moneva, J. \& Cuellar, B. (2009). The value relevance of financial and non-financial environmental reporting. Environmental and Resource Economics, 44(3), 441-456.

Montabon, F., Sroufe, R. \& Narasimhan, R. (2007). An examination of corporate reporting, environmental management practices and firm performance. J. Oper. Manag., 25(5), 998-1014.

Patten, D., Ren, Y. \& Zhao, N. (2015). Standalone Corporate Social Responsibility Reporting in China: An Exploratory Analysis of its Relation to Legitimation. Social and Environmental Accountability Journal, 35(1), 17-31. doi: https://doi.org/10.1080/0969160x.2015.1007467

Russo, M. \& Fouts, P. (1997). A resource-based perspective on corporate environmental performance and profitability. Acad. Manag. J., 40, 534-559.

Silva, M. et al. (2015). Determinantes do disclosure ambiental nos relatórios de empresas listadas na Bovespa. Revista Ambiente Contábil., 7(2), jul./dez.

Simionescu, L. \& Dumitrescu, D. (2018). Empirical Study towards Corporate Social Responsibility Practices and Company Financial Performance. Evidence for Companies Listed on the Bucharest Stock Exchange. Sustainability, 10, 3141.

\section{Notas}

* Pesquisa científica e tecnológica.

\section{Licencia Creative Commons CC BY 4.0}

Para citar este artigo: Silva, M. N., Rodrigues, R. N., \& Lagioia, U. C. T. (2019). É financeiramente viável ser verde? A relação entre as práticas de divulgação e a performance financeira de empresas brasileiras de alto impacto ambiental. Cuadernos de Contabilidad, 20(49). https://doi.org/10.11144/ Javeriana.cc20-49.ffgr 


\section{APENDICE 1}

Categoria Ambiental do GRI G4

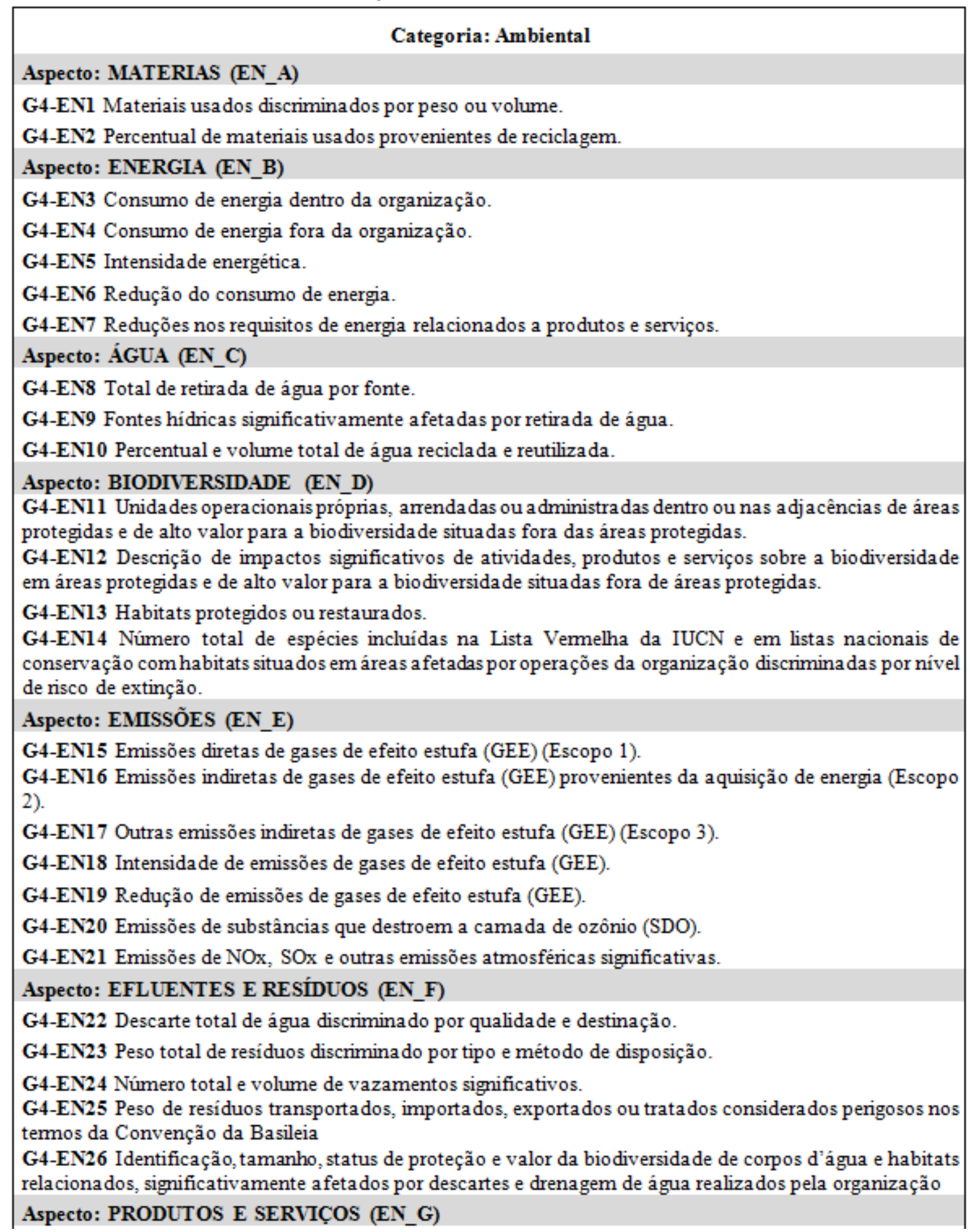


(CONT.)

Categoria Ambiental do GRI G4

G4-EN30 Impactos ambientais significativos decorrentes do transporte de produtos e outros bens e materiais usados nas operações da organização, bem como do transporte de seus empregados.

Aspecto: GERAL (EN_J)

G4-EN31 Total de investimentos e gastos com proteção ambiental discriminado por tipo.

Aspecto: AVALIAC̣Ão AMBIENTAL DE FORNECEDORES (EN_K)

G4-EN32 Percentual de novos fomecedores seleciona dos com base em critérios ambientais.

G4-EN33 Impactos ambientais negativos significativos reais e potenciais na cadeia de fomecedores e medidas tomadas a esse respeito.

Aspecto: MECANISMOS DE QUEIXAS E RECLAMAC̣õES RELACIONADAS A IMPACTOS AMBIENTAIS (EM_L)

G4-EN34 Número de queixas e reclamações relaciona das a impactos ambientais protocola das, processadas e solucionadas por meio de mecanismo formal. 


\section{APENDICE 2}

Correlações entre os indicadores ambientais e os indicadores financeiros

\begin{tabular}{|c|c|c|c|c|}
\hline \multirow{3}{*}{ Taxa de Crescimento das Vendas } & Correlation Coefficient & 1,000 & $-0,216$ & 0,185 \\
\hline & Sig. (1-tailed) & & 0,195 & 0,232 \\
\hline & $\mathrm{N}$ & 18 & 18 & 18 \\
\hline \multirow{3}{*}{ ROE } & Correlation Coefficient & $-0,216$ & 1,000 & 0,027 \\
\hline & Sig. (1-tailed) & 0,195 & & 0,457 \\
\hline & $\mathrm{N}$ & 18 & 18 & 18 \\
\hline \multirow{3}{*}{$\begin{array}{l}\text { Fluxo de caixa operacional / } \\
\text { Receitas }\end{array}$} & Correlation Coefficient & 0,185 & 0,027 & 1,000 \\
\hline & Sig. (1-tailed) & 0,232 & 0,457 & \\
\hline & $\mathrm{N}$ & 18 & 18 & 18 \\
\hline \multirow{3}{*}{ G4-EN3 } & Correlation Coefficient & $-0,070$ & $-0,264$ & 0,007 \\
\hline & Sig. (1-tailed) & 0,391 & 0,145 & 0,489 \\
\hline & $\mathrm{N}$ & 18 & 18 & 18 \\
\hline \multirow{3}{*}{ G4-EN4 } & Correlation Coefficient & 0,051 & 0,392 & $0,540^{\circ}$ \\
\hline & Sig. (1-tailed) & 0,420 & 0,054 & 0,010 \\
\hline & $\mathrm{N}$ & 18 & 18 & 18 \\
\hline \multirow{3}{*}{ G4-EN5 } & Correlation Coefficient & 0,030 & $-0,130$ & 0,088 \\
\hline & Sig. (1-tailed) & 0,453 & 0,303 & 0,364 \\
\hline & $\mathrm{N}$ & 18 & 18 & 18 \\
\hline \multirow{3}{*}{ G4-EN6 } & Correlation Coefficient & 0,172 & 0,071 & 0,192 \\
\hline & Sig. (1-tailed) & 0,247 & 0,390 & 0222 \\
\hline & $\mathrm{N}$ & 18 & 18 & 18 \\
\hline \multirow{3}{*}{ G4-EN7 } & Correlation Coefficient & $-0,211$ & $-0,117$ & 0304 \\
\hline & Sig. (1-tailed) & 0,201 & 0,322 & 0110 \\
\hline & $\mathrm{N}$ & 18 & 18 & 18 \\
\hline \multirow{3}{*}{ G4-EN8 } & Correlation Coefficient & 0,198 & $-0,280$ & -0159 \\
\hline & Sig. (1-tailed) & 0,216 & 0130 & 0264 \\
\hline & $\mathrm{N}$ & 18 & 18 & 18 \\
\hline \multirow{3}{*}{ G4-EN9 } & Correlation Coefficient & $-0,057$ & 0266 & 0105 \\
\hline & Sig. (1-tailed) & 0,412 & 0143 & 0339 \\
\hline & $\mathrm{N}$ & 18 & 18 & 18 \\
\hline \multirow{3}{*}{ G4-EN10 } & Correlation Coefficient & 0,043 & $-0,281$ & -0042 \\
\hline & Sig. (1-tailed) & 0,432 & 0,129 & 0,435 \\
\hline & $\mathrm{N}$ & 18 & 18 & 18 \\
\hline \multirow{3}{*}{ G4-EN11 } & Correlation Coefficient & 0,364 & 0,174 & 0,236 \\
\hline & Sig. (1-tailed) & 0,069 & 0,244 & 0,173 \\
\hline & $\mathrm{N}$ & 18 & 18 & 18 \\
\hline \multirow{3}{*}{ G4-EN12 } & Correlation Coefficient & $0,403^{*}$ & $-0,022$ & 0,319 \\
\hline & Sig. (1-tailed) & 0,049 & 0,465 & 0,098 \\
\hline & $\mathrm{N}$ & 18 & 18 & 18 \\
\hline \multirow{3}{*}{ G4-EN13 } & Correlation Coefficient & 0,122 & 0,089 & $0,427^{*}$ \\
\hline & Sig. (1-tailed) & 0,315 & 0,362 & 0,039 \\
\hline & $\mathrm{N}$ & 18 & 18 & 18 \\
\hline G4-EN14 & Correlation Coefficient & 0,185 & $-0,131$ & 0,345 \\
\hline
\end{tabular}

${ }^{*}$ Correlation is significant at the 0.05 level (1-tailed).

${ }^{* *}$ Correlation is significant at the 0.01 level (1-tailed). 
(CONT.)

Correlações entre os indicadores ambientais e os indicadores financeiros

\begin{tabular}{|c|c|c|c|c|}
\hline & Sig. (1-tailed) & 0,231 & 0,302 & 0,080 \\
\hline & $\mathrm{N}$ & 18 & 18 & 18 \\
\hline & Correlation Coefficient & 0,350 & $-0,608^{*-}$ & 0,002 \\
\hline G4-EN15 & Sig. (1-tailed) & 0,077 & 0,004 & 0,496 \\
\hline & $\mathrm{N}$ & 18 & 18 & 18 \\
\hline & Correlation Coefficient & 0,283 & $-0,522^{*}$ & $-0,112$ \\
\hline G4-EN16 & Sig. (1-tailed) & 0,128 & 0,013 & 0,329 \\
\hline & $\mathrm{N}$ & 18 & 18 & 18 \\
\hline & Correlation Coefficient & 0,292 & $-0,422^{*}$ & 0,073 \\
\hline G4-EN17 & Sig. (1-tailed) & 0,120 & 0,040 & 0,387 \\
\hline & $\mathrm{N}$ & 18 & 18 & 18 \\
\hline & Correlation Coefficient & 0,341 & $-0,436^{*}$ & 0,186 \\
\hline G4-EN18 & Sig. (1-tailed) & 0,083 & 0,035 & 0,230 \\
\hline & $\mathrm{N}$ & 18 & 18 & 18 \\
\hline & Correlation Coefficient & 0,316 & $-0,400$ & 0,143 \\
\hline G4-EN19 & Sig. (1-tailed) & 0,101 & 0,050 & 0,285 \\
\hline & $\mathrm{N}$ & 18 & 18 & 18 \\
\hline & Correlation Coefficient & 0,157 & $-0,291$ & 0,173 \\
\hline G4-EN20 & Sig. (1-tailed) & 0,266 & 0,120 & 0,246 \\
\hline & $\mathrm{N}$ & 18 & 18 & 18 \\
\hline & Correlation Coefficient & 0,398 & $-0,120$ & 0,273 \\
\hline G4-EN21 & Sig. (1-tailed) & 0,051 & 0,318 & 0,136 \\
\hline & $\mathrm{N}$ & 18 & 18 & 18 \\
\hline & Correlation Coefficient & $-0,197$ & $-0,082$ & $-0,231$ \\
\hline G4-EN22 & Sig. (1-tailed) & 0,216 & 0,373 & 0,178 \\
\hline & $\mathrm{N}$ & 18 & 18 & 18 \\
\hline & Correlation Coefficient & 0,055 & $-0,288$ & 0,045 \\
\hline G4-EN23 & Sig. (1-tailed) & 0,414 & 0,123 & 0,430 \\
\hline & $\mathrm{N}$ & 18 & 18 & 18 \\
\hline & Correlation Coefficient & 0,051 & 0,073 & 0,275 \\
\hline G4-EN24 & Sig. (1-tailed) & 0,421 & 0,387 & 0,135 \\
\hline & $\mathrm{N}$ & 18 & 18 & 18 \\
\hline & Correlation Coefficient & 0,185 & 0,124 & 0,375 \\
\hline G4-EN25 & Sig. (1-tailed) & 0,231 & 0,312 & 0,062 \\
\hline & $\mathrm{N}$ & 18 & 18 & 18 \\
\hline & Correlation Coefficient & $-0,107$ & 0,249 & 0,186 \\
\hline G4-EN26 & Sig. (1-tailed) & 0,337 & 0,159 & 0,230 \\
\hline & $\mathrm{N}$ & 18 & 18 & 18 \\
\hline & Correlation Coefficient & 0,342 & $-0,014$ & $-0,041$ \\
\hline G4-EN29 & Sig. (1-tailed) & 0,082 & 0,478 & 0,435 \\
\hline & $\mathrm{N}$ & 18 & 18 & 18 \\
\hline & Correlation Coefficient & $0,514^{*}$ & 0,230 & 0,157 \\
\hline G4-EN30 & Sig. (1-tailed) & 0,015 & 0,179 & 0,267 \\
\hline & $\mathrm{N}$ & 18 & 18 & 18 \\
\hline & Correlation Coefficient & 0,233 & $-0,034$ & 0,256 \\
\hline G4-EN31 & Sig. (1-tailed) & 0,176 & 0,447 & 0,152 \\
\hline & $\mathrm{N}$ & 18 & 18 & 18 \\
\hline & Correlation Coefficient & $0,460^{\circ}$ & $-0,164$ & 0,275 \\
\hline G4-EN34 & Sig. (1-tailed) & 0,027 & 0,258 & 0,135 \\
\hline & $\mathrm{N}$ & 18 & 18 & 18 \\
\hline
\end{tabular}

${ }^{*}$ Correlation is significant at the 0.05 level ( 1 -tailed).

${ }^{* *}$ Correlation is significant at the 0.01 level (1-tailed). 